\title{
Where BRN Reviews is one and a half years after its launch...
}

With this current issue, BRN Reviews has completed, as an independent academic publication, six issues of the Journal since its launch in July 2015. Over the past one and a half years, we have published a number of reviews written by world class experts and have developed an agenda for future production. We still are amazed by the willingness of our authors whose expertise in a number of respiratory medicine areas is acknowledged worldwide, so we are well poised to make further contributions to the ever-growing body of medical publications. Once again we want to underline our immense gratitude for their willingness to collaborate, including the efforts of the reviewers, who all have made possible this important achievement by BRN Reviews.

As we continue to explore new non-traditional avenues for high-quality academic review articles, we hope that BRN Reviews will highlight these achievements and serve as an inspiration for our current and future readers, i.e. respiratory physicians and allied health specialists as they embark on exciting and inspiring personal new projects. We are enthusiastic about the road ahead and look forward to sharing our future breakthroughs with our readers.

What can you read in the current issue? We include herein six articles that we believe will be of great interest to the general reader of the Reviews. The first one by Peter J. Barnes, a top academic personality with great expertise in respiratory medicine research, is devoted to the timely field of dual bronchodilation in patients with chronic obstructive pulmonary disease (COPD). The article describes long-acting bronchodilators as the most effective treatments currently available for the management of patients with stable COPD. Both long-acting muscarinic antagonists and $\beta_{2}$-agonists are equally effective in producing bronchodilation, reducing symptoms, improving quality of life, and preventing exacerbations. Most importantly, these medications are well tolerated and probably work mainly by relaxing smooth 
muscle of peripheral airways to reduce gas trapping. Vital aspects of great interest include modes of action, clinical studies, and side effects.

M. Divo and C. Cabrera present the results from the most influential comorbidities studies in COPD patients, highlighting those that have provided the most relevant information. Moreover, they introduce a novel perspective derived from network analysis, which helps interpret the complex interplay among comorbidities in COPD. This analysis represents a new research tool of great interest. Miguel Divo is an emerging investigator in network analysis who is making important contributions in this field.

The third article by G. Hynes, R. Shrimanker, and I.D. Pavord describes where we are presently with the move towards personalized (precision) medicine in bronchial asthma, firstly explaining why they believe the current medical approach is insufficient, and secondly, outlining in practical terms how physicians can adopt this novel approach when treating patients with bronchial asthma in clinics nowadays. Certainly, this represents a major breakthrough in our understanding of the mechanisms of obstructive airway diseases and, and most importantly, the future for individually customizing their management and therapy. Ian Pavord and his team are making continuous relevant contributions to the mechanistic and therapeutic roles of asthma and COPD.

The fourth review by P.G. Gibson and M. McDonald discusses the clinical, inflammatory, and molecular phenotypes identified in asthma and COPD, and offers an approach to phenotype them that can be implemented in the clinic. In this regard, the concepts of phenotype and endotype are extensively defined. Furthermore, this contribution complements and matches elegantly the previous article as both pave the way of the progressive importance of personalized medicine as a way forward to further identify and treat the various traits of chronic obstructive airway diseases as recently reported in a perspective paper ${ }^{1}$. Peter Gibson and Vanessa McDonald are very active investigators in this field, with continuous innovations in the interpretation of the pathogenesis and management of these obstructive airway disorders.

The fifth paper, by C. Callóniz and A. Torres, focuses on new data in the management of community-acquired pneumonia over the last 15 years. They highlight the explosion of approaches and information from the many studies focusing on community-acquired pneumonia epidemiology, microbial aetiology, and management. Most importantly, using the introduction of predictive tools and advances in their pathobiology, they underline their management and improvement due to the many advantages derived from the implementation of international guidelines. Antoni Torres, a wellknown investigator in pneumonias since the late 1980s, has a renowned background of studies and many influential contributions.

The sixth article by D.E. O'Donnell, N.J. Domnik and J.A. Neder examines exercise pathophysiology in COPD and interstitial lung disease. The authors brilliantly demonstrate 
striking similarities in the physiological responses to exercise across these two pathologically distinct disorders, and provide evidence to support common underlying mechanisms of exertional dyspnoea and reduced exercise capacity. Likewise, they discuss the clinical implications of these new advances in exercise pathophysiology in the context of targeted therapeutic interventions. Dennis O'Donnell and his associates are creatively enriching this area of exercise research by combining measures of symptom intensity, metabolic and ventilatory control outcomes, as well as dynamic respiratory mechanics during standardized incremental tests to tolerance.
On behalf of the BRN Reviews editorial team, please follow our progress and help celebrate our accomplishments by reading our BRN Reviews issues. Thank you in advance for your support and interest in BRN Reviews. We hope and expect that you will enjoy and learn from this new issue.

\section{R. Rodriguez-Roisin} September 2016

\section{REFERENCES}

1. Agusti A, Bel E, Thomas M, et al. Treatable traits: toward precision medicine of chronic airway diseases. Eur Respir J. 2016;47:410-9. 